\title{
Cryopreservation Benefits in Cryptozoospermia, Severe Oligozoospermia and Non-Obstructive Azoospermia in Male Infertility
}

\author{
Aya Al-Ibraheemi ${ }^{1}$, Noureddine Louanjli ${ }^{2}$, Mohamed Zarqaoui ${ }^{3}$, Mohamed Ennaji ${ }^{3}$, \\ Wassym R. Senhaji ${ }^{3}$, Ritu S. Santwani ${ }^{4}$, Nisrine En-Naciri ${ }^{5}$, Hafida Tarik ${ }^{5}$, Romaissa Boutiche ${ }^{6}$, \\ Mustafa Zakaria ${ }^{3 *}$
}

${ }^{1}$ ART IRIFIV Scientific Research Group (AISRG), Casablanca, Morocco

${ }^{2}$ LABOMAC Laboratory, and IRIFIV Fertility Center, AFC Fertility Center, Casablanca, Morocco

${ }^{3}$ The Society for ART IRIFIV Scientific Research Group (IRIFIV-AISRG), Casablanca, Morocco

${ }^{4}$ India Society for Assisted Reproduction (ISAR), Garjula, India

${ }^{5}$ Laboratory IVF Agadir, ART IRIFIV Scientific Research Group (AISRG), Agadir, Morocco

${ }^{6}$ Laboratory IVF Algeria, Rotary Fertility Center, Algiers, Algeria

Email: ^dr.zakaria@irifiv-aisrg.com

How to cite this paper: Al-Ibraheemi, A., Louanjli, N., Zarqaoui, M., Ennaji, M., Senhaji, W.R., Santwani, R.S., En-Naciri, N., Tarik, H., Boutiche, R. and Zakaria, M. (2021) Cryopreservation Benefits in Cryptozoospermia, Severe Oligozoospermia and Non-Obstructive Azoospermia in Male Infertility. Open Access Library Journal, 8: e7190.

https://doi.org/10.4236/oalib.1107190

Received: January 27, 2021

Accepted: February 23, 2021

Published: February 26, 2021

Copyright () 2021 by author(s) and Open Access Library Inc.

This work is licensed under the Creative Commons Attribution International License (CC BY 4.0).

http://creativecommons.org/licenses/by/4.0/

\begin{abstract}
Male infertility counts for almost $30 \%$ of the total cases of infertility. Male infertility can be caused by several and various factors such as environmental, genetic and hormonal factors. Male infertility can be diagnosed through semen sample analysis; a fresh semen sample can indicate different semen abnormalities. A semen sample can indicate Azoospermia, Cryptozoospermia (Co) or Severe Oligozoospermia (ESO), which can cause infertility in male. It is challenging for an andrologist to distinguish between ESO and CO in clinic diagnose since both show no sperm in the sample in the initial sample test. In Cryptozoospermia or Severe Oligozoospermia, cryopreservation plays a significant part in preserving male fertility by freezing the individual sperm.
\end{abstract}

\section{Subject Areas}

Andrology

\section{Keywords}

Male Infertility, Semen Analysis, Abnormal Semen Sample, Cryptozoospermia and Cryopreservation

${ }^{*}$ Corresponding author. 


\section{Introduction}

\subsection{Infertility}

Infertility is the failure to conceive or get pregnant within a sensible trying period, usually 6 to 12 months. Sterility is a complete failure to develop pregnancy, which can be reversible or irreversible. Subfertility is infertility without an absolute limit to reproduction which can cause sterility, such as azoospermia. Infertility can be categorised into three categories, female infertility, male infertility, and unknown factors infertility [1]. Female infertility counts for approximately $30 \%$, including anovulation, tubal obstruction, or other pathology, where male infertility disorder counts about $30 \%$, and conditions in both partners in $30 \%$. No abnormalities are found in approximately $10 \%$ (Miyamoto et al., 2012) [2].

\subsection{Male Infertility}

Male infertility is described as the incapability of a male to fertilize female oocytes and make a fertile female pregnant for at least one year of unprotected intercourse. Several factors can cause male infertility, such as genetics and environmental factors. Environmental factors can be produced by exposure to environmental toxins, obesity, and age (Leslie SW et al., 2020) [3]; the below diagram illustrates most male infertility factors (Figure 1).

The environmental factors associated with male infertility have been studied and examined in several types of research, where they have found that men regularly are exposed to hazardous materials in their daily work such as solvents, pesticides, adhesives, silicones and radioactivity, exposure to these and related substances can be a potential cause of male infertility. Radiation exposure can reduce sperm production, and susceptibility to high dosages can cause complete infertility. Additionally, the overuse of the sunbath can likewise lead to a temporary drop in sperm count. Besides, jobs which need long-time of sitting (such as driving) or remaining exposed to high temperatures (such as bakeries) can

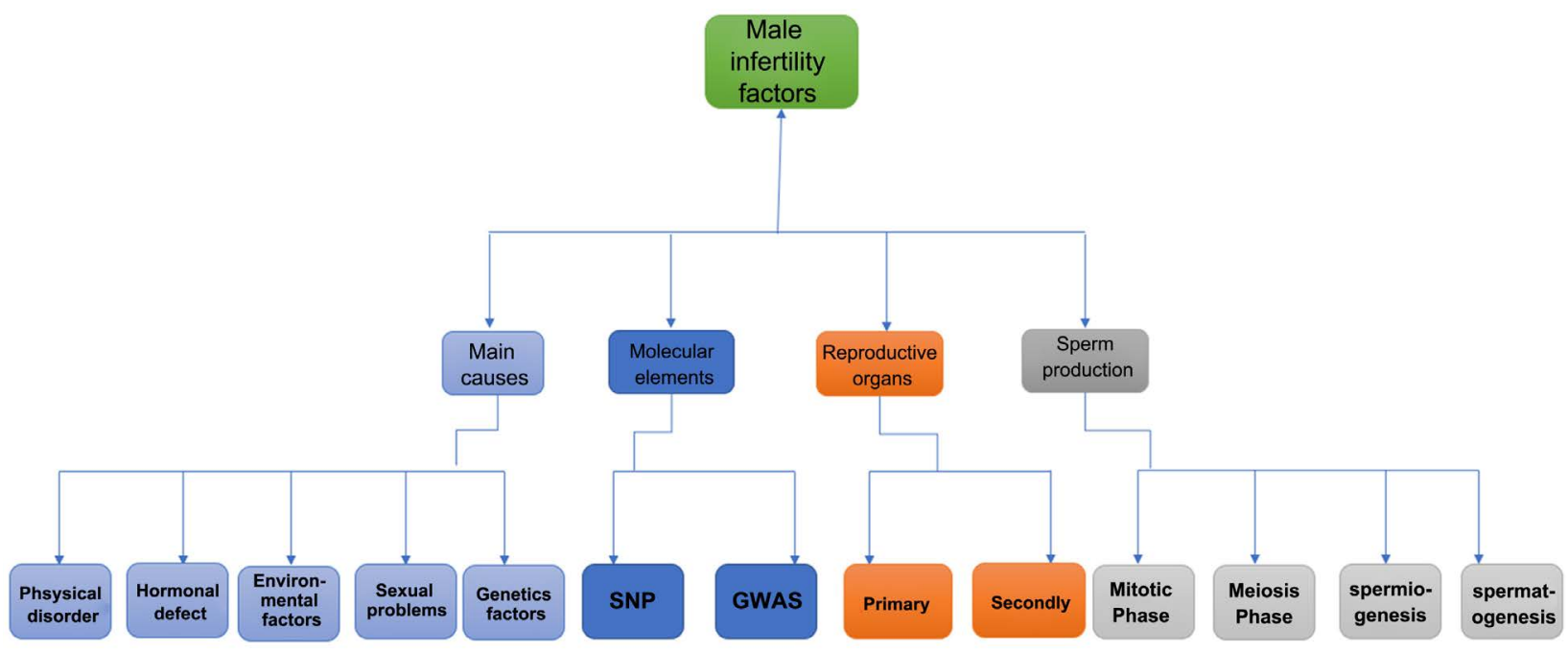

Figure 1. An illustrative flow-map of the main factors that can lead to male infertility (Babakhanzadeh, et al., 2012) [1]. 
negatively influence fertility. There is no faultless understanding of their sperm parameters and fertility outcomes regarding alcohol consumption and smoking. Nevertheless, increasing sperm degradation quality may be linked to cigarette smoking and alcohol consumption. Inadequate nutrition can also play a part in male infertility. Men are usually seen to be protected against age fertility problems. However, assisted reproductive technologies started to raise awareness about the advanced paternal age (APA). Besides, APA has also been linked with numerous offspring sicknesses like achondroplasia, autism, schizophrenia, and bipolar disorders (Sharma et al., 2015) [4]. Firstly, many studies illustrate the old paternal age and its disadvantageous impacts on sperm quality and parameter [5]. In one study about sperm count and sperm motility in aged rats, it has been found that sperm count was fallen notably in rat female's uterus at 24 months of age, due to a reduction in sperm production. Also, the prostate gland and dorsal prostatic secretions, which are required for sperm motility, had morphological prostate alterations and secretion modification at ageing rats Also, the study results showed a link between sperm immobility and motility and a failure in the prostatic function in old male rats when sperm motility was decreased at both 12 months, 24 months of age (Lucio et al., 2013) [6] (Figure 2).

Moreover, any medicine, tumour, illness, or disorder that influences the pituitary gland or hypothalamus could be a potential cause of male infertility through modifying gonadotropic releasing hormone or producing gonadotropin as idiopathic hypogonadotropic hypogonadism (IHH), Kallmann syndrome (IHH with anosmia), and mixed pituitary hormone deficiency (Leslie SW et al., 2020) [3]. Pituitary tumours like sellar tumours, macroadenomas, and prolactinomas can also rise in male infertility due to changes in gonadotropin hormone production and several genetic causes Prader-Willi, Young, and Laurence-Moon-Biedl syndromes, can lead to male infertility. Many acquired disorders, such as primary androgen overproduction and exogenous testosterone supplementation, would also instantly reduce gonadotropic secretion inducing decreased sperm counts and infertility (Leslie SW et al., 2020) [3].

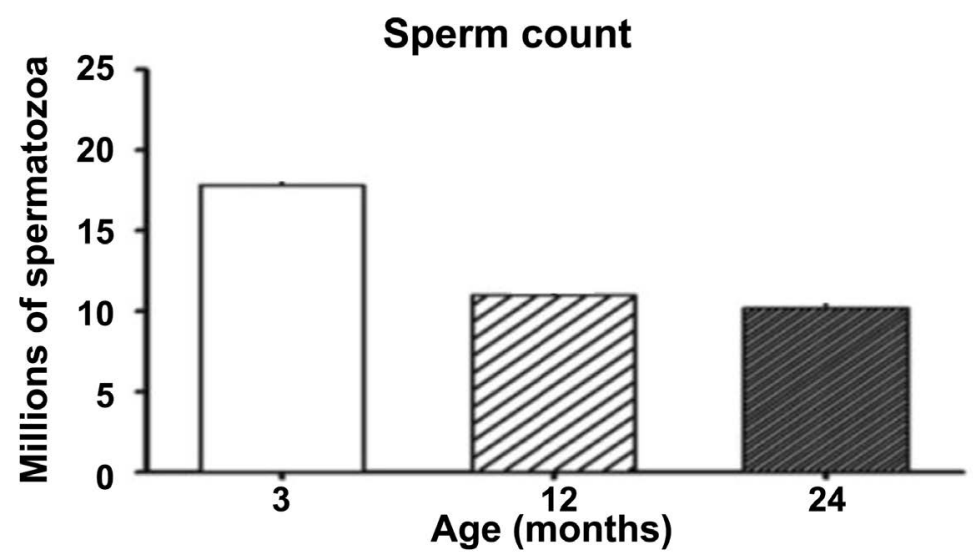

Figure 2. Sperm count alteration in aging rat; the diagram illustrate that sperm count is decaling with increasing rat age (Lucio et al., 2013) [6]. 


\section{Semen Analysis and Male Infertility}

Semen has two main elements: the spermatozoa produced by the testis' seminiferous tubules. The seminal fluid made at the accessory glands supports sperm and has a premium role in interacting with the female reproductive tract to induce fertilization. These elements are shown in the semen analysis through the sperm count, that indicates the number of spermatozoa in the semen sample; and the volume of the semen, which reveals the amount of seminal fluid produced. Sperm motility is described as the rate of sperm that moves through the seminal fluid, while the sperm morphology is the percentage of sperm with a standard cellular structure (Patel, Leong and Ramasamy, 2018) [7]. For accurate results, the semen sample is obtained by masturbation after an abstinence period of $2-7$ days. The semen sample has some physical characteristics which can be measured, like the volume, $\mathrm{pH}$, colour, liquefaction, and viscosity. The sample is then assessed through a microscope to define the motility, vitality, concentration, and morphology. The semen analysis results are compared to the reference values determined by the WHO manual (Patel, Leong and Ramasamy, 2018) [7].

\section{Abnormal Semen Sample}

The semen analysis can indicate several and various semen sample abnormalities. The abnormalities can be related to concentration, morphology, and motility of the sperms (Table 1). For instance, Azoospermia is a condition where spermatozoa are absent in the sediment of a centrifuged semen sample of a man, where cryptozoospermia is an abnormal semen condition deficient spermatozoa concentration $(\leq 1 \mathrm{million} / \mathrm{mL})$ in the ejaculate of a man. These circumstances are usually diagnosed through a routine male infertility examination (Cocuzza, Alvarenga and Pagani, 2013) [8].

As discussed above, Cryptozoospermia (CO) is a condition described by the World Health Organization where spermatozoa cannot be recognized or found in a fresh semen sample. Still, it can be observed with extended centrifugation

Table 1. The WHO categorization of an abnormal semen sample (WHO, 2010).

\begin{tabular}{ll}
\hline \multicolumn{1}{c}{ Term } & \multicolumn{1}{c}{ Definitions } \\
\hline Normozoospermia & $\begin{array}{l}\text { Normal ejaculate as defined by the reference values } \\
\text { Oligozoospermia }\end{array}$ \\
Sperm concentration fewer than $20 \times 10^{6} / \mathrm{mL}$ \\
Asthenozoospermia & $\begin{array}{l}\text { Few than } 50 \% \text { spermatozoa with forward progression (categories } \\
\text { movement }\end{array}$ \\
Teratozoospermia & $\begin{array}{l}\text { Fewer than } 30 \% \text { spermatozoa with normal morphology Less than } \\
\text { the reference value for morphology }\end{array}$ \\
Oligoasthenoteratozoospermia & $\begin{array}{l}\text { Signifies disturbance of all three variables (combinations of only } \\
\text { two prefixes may also be used) }\end{array}$ \\
Azoospermia & No spermatozoa in the ejaculate \\
Aspermia & No ejaculate
\end{tabular}


and microscopic process. However, the spermatozoa found in a section of semen samples using a microscopic directly, but cannot be included due to low concentration; this condition is diagnosed as a severe oligozoospermia (ESO) (Deng et al., 2015) [9]. ESO that the World Health Organization has not described explicitly states that spermatozoa concentration is $<1 \times 10^{6} \mathrm{ml}^{-1}$, based on the definition of severe oligoasthenoteratozoospermia by various studies. ESO and CO patients are advised to go for intracytoplasmic sperm injection (ICSI) as infertility treatment as their partners are challenging to get pregnant easily or by drug treatment. ICSI assists them to be biological parents by using their single spermatozoa that have been collected from ejaculation or percutaneous epididymal sperm aspiration (PESA) or testicular sperm aspiration (TESA), even testicular sperm extraction (TESE) or micro-TESE. CO can be influenced by environment temperature, the atmosphere of the masturbation room, the ejaculation state, induction by audio-visual devices, etc. In cryptozoospermia, the sperm count fluctuates remarkably in sperm count, pointing to challenges in obtaining sperm in the ejaculate on the day of oocyte retrieval (Koscinski et al., 2013) [10]. Thus $\mathrm{CO}$ is usually thought to be virtual azoospermia and regularly needs testicular sperm extraction (TESE) for intracytoplasmic sperm injection (ICSI) treatment. Additionally, $\mathrm{CO}$ can cause various risks to male infertility treatment; for instance, possible andrological risks are linked to testicular biopsies, such as vascular injuries and additional androgenic defect or testicular atrophy; the utility of testicular gametes, that can be less mature from the ejaculated spermatozoa. Therefore, an extended sperm preparation (ESP) protocol should be applied regularly to confirm the accurate diagnostic of $\mathrm{CO}$ and azoospermia, particularly in the absence of obstructive aetiology (Koscinski et al., 2013) [10]. On the other hand, oligozoospermia is frequently linked with exogenous factors like infectious agents, environmental pollutants, age, and obesity. Male with oligozoospermia does have few sperm, more than those with azoospermia or CO, although significantly fewer than normal fertile male. Furthermore, this condition is associated with irregular sperms morphology (Badal, 2017) [5]. When oligozoospermia developed to a severe case, it's then called severe oligozoospermia (ESO), and it's equal to Cryptozoospermia. Both CO and ESO can be caused by genetic abnormalities, medical history and abnormal physical examination. For instance, an abnormal karyotype in 47, XXY and hypogonadism can cause CO; deletion of the azoospermia factor (AZF) region and hypogonadism can also cause $\mathrm{CO}$ and azoospermia (Dohle, 2002) [11]. Still, ESO and CO are not etiologist representative, but only an analysis of semen parameters. Hence, in some cases, patients can Have testicular dysfunction like Y chromosome microdeletion, and others may have incomplete seminal tract obstruction conditions. At the same time, their spermatogenesis is normal as OA. This can create heterogeneity of patients in ESO and CO Groups. In another case study, 33-years old patient had to go for sterility treatment for almost five years. A novel homozygous nonsense mutation has been found through the diagnostic journey, which was associated with cryptozoospermia. This mutation can also be a genetic factor of cryptozoospermia, 
with TEX15 deficiency causing spermatogenesis failure. (6) The mutation was inherited from the patient's parents, who were both heterozygous carriers, representative of a recessive pattern of inheritance, and the development of effective medicine, making TEX15 deficiency a clinical marker for recognition of nonobstructive azoospermia or cryptozoospermia (Sha et al., 2018) [12]. The treatment main treatment option for male infertility in cases of CO and ESO is ICSI. ICSI was a transformation method that avoided difficulties in natural sperm and oocyte fertilization and fusion, particularly appropriate for severing oligozoospermia and cryptozoospermia. Although, if no sperm can be obtained on the day of oocyte retrieval, it can lead to the cancellation of ICSI (Deng et al., 2015) [9]. Thus, the testis biopsy can be the gold standard in such cases to assess spermatogenesis, which is not a routine investigation in CO and ESO. Consequently, it is essential to evaluate spermatogenic functions in patients with $\mathrm{CO}$ and ESO, through the volume of testis size, FSH levels, and inhibin B in advance. Additionally, it is prefeed to obtain a testicular if CO and ESO were accompanied with azoospermia (Deng et al., 2015) [9]. Also, To avoid ICSI cancellation on the day of oocytes retrieval, most IVF centres offer substitute options such as surgically sperm extraction (PESA or TESE) to synchronize oocyte retrieval (Ketabchi, 2016) [13].

\section{Cryptozoospermia and Cryopreservation}

Cryopreservation of human sperms has been applied in the 1960s, where it has been known to be an effective method of the male fertility management before treatment for malignant diseases, vasectomy or surgical infertility treatments, to save donor and partner spermatozoa before assisted reproduction procedures and to assure the recovery of a small number of spermatozoa in severe male factor infertility, such as in the cases of the cryptozoospermia or severe oligospermia (Di Santo, Tarozzi, Nadalini and Borini, 2012). The traditional approaches of sperm cryopreservation cannot be applied to freeze small numbers of cells, like epididymal and testicular spermatozoa. Thus effective and novel cryopreservation of surgically gained spermatozoa, can decrease the number of surgical interventions and reduced the logistic risks associated with managing the women's oocyte retrieval, additionally to the risk of no sperm can be gained on the same day of oocyte retrieval (Di Santo, Tarozzi, Nadalini and Borini, 2012). Conventional sperm cryopreservation methods can lead to sperm loss through sperm adherence to the carrier vessel, inadequate centrifugation, and washing phase. Therefore, the traditional approach is especially problematic in severe oligospermia and cryptozoospermia (Liu and $\mathrm{Li}, 2020$ ) [14]. A novel cryopreservation method for few sperms utilises an empty zona pellucida (ZP) by providing theoretical and technical support for other procedures. After introducing such a single-sperm freezing technique, several cryopreservation methods have been introduced to enhance sperm counts ( $\mathrm{Liu}$ and $\mathrm{Li}, 2020$ ). Similar to the non-biological empty ZP method, an empty capsule of a non-biological carrier has been developed which is received from an animal, where small spermatozoa 
have been frozen in polymerised alginic acid drops. Alginic acid is an inert chemical of two sugars mixture, namely, $\beta$-D-mannuronic and $\alpha$-L-guluronic acid. Alginate capsules carrying sperm are stored in the cryoprotective agent (CPA) and preserved in straws using a programmable freezer. Upon thawing, alginate capsules are dissolved in a sodium citrate solution [2]. The advantages of using alginate are that it is a non-hazardous polysaccharide; it can be applied successfully for the cryopreservation of hepatocytes and stem cells; it has liquid gel characteristics inertness chemical. However, cryopreservation of human sperm through this procedure produced a $20 \%$ reduction in motility compared with standard protocols. It is proposed that this is possibly caused by the alginic acid covering the surface of the sperm. Due to the complexity of the complete operation and other shortcomings, this technology's developments are slow, limiting its clinical application (Liu and Li, 2020) [14]. Another effective method applied for cryptozoospermia cryopreservation is called cell sleeper, which is a closed cryopreservation system. The Cell Sleeper has a vial container implemented with an internal tray, where individual sperm can be added to a droplet $(3.5 \mu \mathrm{L})$ on the tray. Following, the tray is placed into a vial and covered by a screw cap. The vial is then put in $\mathrm{LN} 2$ vapour $\left(-120^{\circ} \mathrm{C}\right)$ before the exposure to sterilised LN2 [7].

\section{Conclusion}

In conclusion, male infertility is common and can account for a high percentage of infertility disorder. CO and ESO are complex male infertility disorder, which can be caused by several factors such as genetics and hormonal factors. Therefore, sperms left in the male reproductive system in CO and ESO cases can be the last hope key for numerous partners; thus, they need to be collected and treated in a well organised and effective way [12]. Cryopreservation would be the best CO and ESO options to preserve individual sperms; still, a well-developed protocol and techniques should be used to protect those individual sperms.

\section{Acknowledgements}

The Objective of the Society for Art Irifiv Science Research Group (IRIFIV-AISRG), Research foundation in Casablanca, Maintaining consistent and reliably high success rates is a monthly challenge for in IVF labs, the IRIFIV Fertility Center in Casablanca-Morocco Department of Reproductive Medicine and Reproductive Biology and Embryology, advocacy of interdisciplinary Department of Reproductive Medicine and Reproductive Biology and Embryology study, encompassing the areas of research, collections and publishing Articles.

\section{Conflicts of Interest}

The authors declare no conflicts of interest regarding the publication of this paper. 


\section{References}

[1] Babakhanzadeh, E., Nazari, M., Ghasemifar, S. and Khodadadian, A. (2020) Some of the Factors Involved in Male Infertility: A Prospective Review. International Journal of General Medicine, 13, 29-41. https://doi.org/10.2147/IJGM.S241099

[2] Miyamoto, T., Tsujimura, A., Miyagawa, Y., Koh, E., Namiki, M. and Sengoku, K. (2012) Male Infertility and Its Causes in Human. Advances in Urology, 2012, Article ID: 384520. https://doi.org/10.1155/2012/384520

[3] Leslie, S.W., Siref, L.E., Khan, M.A.B. and Male Infertility (2020). Male Infertility. StatPearls Publishing, Treasure Island, FL.

[4] Sharma, R., Agarwal, A., Rohra, V., Assidi, M., Abu-Elmagd, M. and Turki, R. (2015).Effects of Increased Paternal Age on Sperm Quality, Reproductive Outcome and Associated Epigenetic Risks to Offspring. Reproductive Biology and Endocrinology, 13, Article Number: 35. https://doi.org/10.1186/s12958-015-0028-x

[5] Bakare, T., Ghayda, R., Abhyankar, N., Shoshany, O. and Niederberger, C. (2017) Cryptozoospermia. Fertility and Sterility, 108, e313-e314. https://doi.org/10.1016/j.fertnstert.2017.07.927

[6] Lucio, R., Tlachi-López, J., Eguibar, J. and Ågmo, A. (2013) Sperm Count and Sperm Motility Decrease in Old Rats. Physiology \& Behavior, 110-111, 73-79. https://doi.org/10.1016/j.physbeh.2012.12.015

[7] Patel, A., Leong, J. and Ramasamy, R. (2018) Prediction of Male Infertility by the World Health Organization Laboratory Manual for Assessment of Semen Analysis: A Systematic Review. Arab Journal of Urology, 16, 96-102. https://doi.org/10.1016/j.aju.2017.10.005

[8] Cocuzza, M., Alvarenga, C. and Pagani, R (2013) The Epidemiology and etIology of Azoospermia. Clinics, 68, 15-26. https://doi.org/10.6061/clinics/2013(Sup01)03

[9] Deng, Y., Chu, Q., Zhu, Y., Luo, C., Li, Y., Li, H., Quan, S., Yang, Y., Hu, Y. and Tan, W. (2016) Differences and Similarities between Extremely Severe Oligozoospermia and Cryptozoospermia in Intracytoplasmic Sperm Injection. Asian Journal of Andrology, 18, 904-907.

[10] Koscinski, I., Viville, S., Lefebvre-Kahlil, V., Defossez, A. and Rigot, J. (2013) Pregnancies in Cryptozoospermia with Sperm Ejaculated One Day before ICSI: Four Case Reports. Journal of Andrology, 28, 15-20. https://doi.org/10.2164/jandrol.106.000158

[11] Dohle, G. (2002) Genetic Risk Factors in Infertile Men with Severe Oligozoospermia and Azoospermia. Human Reproduction, 17, 13-16.

https://doi.org/10.1093/humrep/17.1.13

[12] Sha, Y., Gao, Z., Wang, X., Jin, H., Cui, Y. and Chen, J. (2018) Case Study of a Patient with Cryptozoospermia Associated with a Recessive TEX15 Nonsense Mutation. Asian Journal of Andrology, 20, 101. https://doi.org/10.4103/1008-682X.194998

[13] Ketabchi, A. (2016) Intracytoplasmic Sperm Injection Outcomes with Freshly Ejaculated Sperms and Testicular or Epididymal Sperm Extraction in Patients with Idiopathic Cryptozoospermia. Nephro-Urology Monthly, 8, e41375. https://doi.org/10.5812/numonthly.41375

[14] Liu, S. and Li, F. (2020) Cryopreservation of Single-Sperm: Where Are We Today? Reproductive Biology and Endocrinology, 18, Article Number: 41. https://doi.org/10.1186/s12958-020-00607-x 


\section{Abbreviations}

Intracytoplasmic sperm injection (ICSI), Advanced paternal age (APA), Testicular sperm aspiration (TESA), Percutaneous epididymal sperm aspiration (PESA), Zona pellucida (ZP), Cryoprotective agent (CPA), Azoospermia factor (AZF), Extended sperm preparation (ESP), Follicle-stimulating hormone (FSH), Cryptozoospermia (CO), Extremely severe oligozoospermia (ESO), Liquid nitrogen (LN2). 